\title{
Coronary Vasospasm-Induced Myocardial Infarction: An Uncommon Presentation of Unrecognized Hyperthyroidism
}

\author{
Basma Ataallah ${ }^{\mathrm{a}, \mathrm{d}}$, Barjinder Buttar ${ }^{\mathrm{a}}$, Alan Kaell ${ }^{\mathrm{a}}$, \\ Georgia Kulina $^{\mathrm{b}}$, Robert Kulina ${ }^{\mathrm{c}}$
}

\begin{abstract}
Hyperthyroidism can present with cardiac issues, such as tachycardia, atrial fibrillation, and high output congestive heart failure. Rare case reports of coronary vasospasm leading to myocardial infarction (MI) are published. Of these cases, many are known to be hyperthyroid prior to cardiac presentation. We report a female patient with unrecognized thyrotoxicosis who presents with acute MI secondary to coronary vasospasm.
\end{abstract}

Keywords: Endocrinology; Hyperthyroidism; Thyrotoxicosis; Coronary vasospasm; Thyroid disease; Graves' disease; Myocardial infarction

\section{Introduction}

Hyperthyroidism has multiple cardiac implications: increases in heart rate, contractility, cardiac output, systolic pressure, mean pulmonary artery pressure, and myocardial oxygen consumption [1]. Untreated or unrecognized hyperthyroidism can manifest as atrial fibrillation or congestive heart failure. Coronary artery vasospasm tends to occur in patients less than 50 years old with Graves' disease and can be treated with antithyroid medications and calcium channel blockers [1, 2]. Acute myocardial infarction (MI) attributed to vasospasm is rarely seen as a presentation of hyperthyroidism [3].

\section{Case Report}

A 47-year-old anxious Hispanic woman presented to the emer-

Manuscript submitted April 18, 2020, accepted April 24, 2020

aDepartment of Internal Medicine, Donald and Barbara Zucker School of Medicine at Hofstra/Northwell, Mather Hospital, Port Jefferson, NY 11777, USA ${ }^{b}$ Department of Endocrinology, Donald and Barbara Zucker School of Medicine at Hofstra/Northwell, Mather Hospital, Port Jefferson, NY 11777, USA ${ }^{\mathrm{c}}$ Department of Cardiology, Stony Brook University School of Medicine, Stony Brook, NY, USA

${ }^{\mathrm{d} C}$ Corresponding Author: Basma Ataallah, Department of Internal Medicine, Donald and Barbara Zucker School of Medicine at Hofstra/Northwell, Mather Hospital, Port Jefferson, NY 11777, USA. Email: bataallah@northwell.edu

doi: https://doi.org/10.14740/jmc3473 gency department with a 1-week history of severe, intermittent, stabbing substernal chest pain radiating to the left arm occurring at rest, but more pronounced on exertion with associated palpitations and shortness of breath. Vital signs showed that the patient was afebrile, heart rate was $100 \mathrm{bpm}$, regular, and blood pressure (BP) was 120/60 mm Hg. A fine tremor and brisk reflexes were noted without tender thyroid, lid lag or proptosis.

Initial electrocardiogram showed: T-wave inversions in leads V1 - V2 and ST depression in V4 - V5; chest pain, immediately relieved by sublingual nitroglycerin followed by dynamic electrocardiography (EKG) changes in varying leads during subsequent episodes of chest pain. Echocardiogram was negative for wall motion abnormalities. Laboratory results revealed troponin elevated to $0.20 \mathrm{ng} / \mathrm{mL}$ (normal level $<0.06$ $\mathrm{ng} / \mathrm{mL}$ ) and findings that were consistent with Graves' disease (Table 1).

Thyroid ultrasound was done and showed heterogeneously enlarged gland with increased vascularity and nodularity.

As for hospital course, a confirmed non-ST-elevation MI (NSTEMI) was managed with heparin drip, aspirin, clopidogrel, high-intensity statin, beta blocker (propranolol for hyperthyroidism), isosorbide mononitrate, and sublingual nitroglycerin. Methimazole was used to treat her hyperthyroidism that was later confirmed as Graves' disease with positive thyroidstimulating immunoglobulin (TSI) and thyroid-stimulating hormone receptor antibodies (TRAbs). Once the patient was adequately loaded with methimazole, cardiac catheterization was performed 3 days later. Angiography demonstrated severe spontaneous coronary vasospastic changes with mild non-obstructive coronary artery disease. Diltiazem was added after confirming coronary vasospastic changes. Patient symptoms were improved and she was discharged home after 5 days. A follow-up at 6 months after discharge revealed improvement in her thyroid function test with thyroid-stimulating hormone (TSH) of 0.676 and free thyroxine (FT4) of 1.11 .

\section{Discussion}

Spontaneously detected vasospasm is one factor in our patient's NSTEMI [3]. The potential pathophysiology of vasospasm and myocardial ischemia in our patient with non-obstructive coronary arteries is unclear and includes direct metabolic effect 
Table 1. Laboratory Results

\begin{tabular}{llllll}
\hline TSH 0.01 mU/L & Total T4 22.26 $\mu \mathrm{g} / \mathrm{dL}$ & Free T4 5.54 & Free T3 21.0 ng/mL & TRAb 7.47 IU/L & TSI 3.61 IU/L \\
$(\mathrm{LLN}>0.45 \mathrm{mU} / \mathrm{L})$ & $(\mathrm{ULN} 12.23 \mu \mathrm{g} / \mathrm{dL})$ & $(\mathrm{ULN}<1.46 \mathrm{ng} / \mathrm{dL})$ & $(\mathrm{ULN}<4.4 \mathrm{pg} / \mathrm{mL})$ & $(\mathrm{ULN}<1.75)$ & $(\mathrm{ULN}<0.55 \mathrm{IU} / \mathrm{L})$ \\
\hline
\end{tabular}

TSH: thyroid-stimulating hormone; T4: thyroxine; T3: triiodothyronine; TRAb: TSH receptor antibody; TSI: thyroid-stimulating immunoglobulin; ULN: upper level of normal; LLN: lower limit of normal.

of thyroid hormone on the myocardium with hypersensitivity to vasoconstrictive hormones leading to a mismatch of blood supply and oxygen demand. Additionally, in-situ coronary thrombosis secondary to vasospasm and stasis of blood can lead to ischemia [3].

Should our patient be considered as having a type 2 MI and/or MI with non-obstructive coronary artery disease, known as MINOCA [4]? Depending upon study design and inclusion/exclusion criteria this occurs more in young women with acute MI. The Variation in Recovery: Role of Gender on Outcomes of Young AMI Patients (VIRGO) study compared the clinical characteristics and outcome of young patients with MINOCA versus obstructive type myocardial infarction (MICAD). MINOCA versus MI-CAD analysis found respectively: $11.1 \%$ had MINOCA versus $88.4 \%$ had MI-CAD, $0.6 \%$ remain unclassified, $6.7 \%$ had unspecified thyroid disease in the MI-CAD group versus $9.7 \%$ in the MINOCA group, and $7.1 \%$ were Hispanic in MI-CAD group versus $10.4 \%$ in MINOCA. Presentation with MINOCA in this study sample was found to be more common in younger nonwhite women and additionally, patients with MINOCA had similar outcomes, including mortality and psychosocial and functional status, to MI-CAD patients [5].

Hyperthyroidism is not listed as a specific exclusion [4] and may not be recognized prior to chest pain presentation. Does our patient meet MINOCA criteria [6]? As type 2 MI and MINOCA are diagnosed post-angiography, perhaps screening for hyperthyroidism, a diagnosable and treatable condition, should be considered in MI patients.

\section{Conclusions}

Unrecognized hyperthyroidism can occur in a patient with an MI. Once both are managed, angiography may reveal nonobstructive coronary disease with or without vasospasm. If spontaneous vasospasm is noted, diltiazem is recommended. Perhaps consensus criteria developers should consider whether unrecognized or inadequately treated hyperthyroidism be explicitly listed as exclusion for MINOCA.

\section{Acknowledgments}

None to declare.

\section{Financial Disclosure}

None to declare.

\section{Conflict of Interest}

None to declare.

\section{Informed Consent}

Not applicable.

\section{Author Contributions}

BA was the first author, collected data, guided the literature search, and wrote the manuscript; BB helped collecting data and writing the article. AK, GK and RK reviewed and supervised the article.

\section{Data Availability}

The authors declare that data supporting the findings of this study are available within the article.

\section{References}

1. Klein I, Ross DS, Mulder JE. Cardiovascular effects of hyperthyroidism. 2019. https://www.uptodate.com/contents/ cardiovascular-effects-of-hyperthyroidism\#H3253683.

2. Choi YH, Chung JH, Bae SW, Lee WH, Jeong EM, Kang $\mathrm{MG}, \mathrm{Kim}$ BJ, et al. Severe coronary artery spasm can be associated with hyperthyroidism. Coron Artery Dis. 2005;16(3):135-139.

3. Kuang XH, Zhang SY. Hyperthyroidism-associated coronary spasm: A case of non-ST segment elevation myocardial infarction with thyrotoxicosis. J Geriatr Cardiol. 2011;8(4):258-259.

4. Thygesen K, Alpert JS, Jaffe AS, Chaitman BR, Bax JJ, Morrow DA, White HD, et al. Fourth universal definition of myocardial infarction (2018). J Am Coll Cardiol. 2018;72(18):2231-2264.

5. Safdar B, Spatz ES, Dreyer RP, Beltrame JF, Lichtman JH, Spertus JA, Reynolds HR, et al. Presentation, clinical profile, and prognosis of young patients with Myocardial Infarction with Nonobstructive Coronary Arteries (MINOCA): results from the VIRGO study. J Am Heart Assoc. 2018;7(13):e009174.

6. Alpert JS, Serpytis R, Serpytis P, Chen QM. Myocardial Infarction with Nonobstructive Coronary Arteries (MINOCA). Am J Med. 2019;132(3):267-268. 